\title{
LA DISCUSIÓN DE LO FANTÁSTICO EN EUROPA DEL SIGLO XX
}

\begin{abstract}
Resumen: La tradición en dar un marco teórico a lo fantástico tiene en la literatura una larga trayectoria así que ya en la edad antigua tenemos reflexiones sobre el tema hechas por Aristóteles y Platón. Se puede decir que con los trabajos de Tzvetan Todorov llega la tradición del marco teórico a un punto clave, es decir todas las teorías antiguas llevan a la de Todorov y todas las nuevas se derivan de la misma. En el ámbito de habla alemán y francés se observa desde entonces una discusión viva con el objetivo dar un marco teórico a lo fantástico en la literatura y para poder luego definir la literatura fantástica que tuvo su auge en Alemania a fin de siglo XIX. La contribución de Marianne Wünsch, que se discute y presente a continuación, aporta a la discusión lo esencial para abrir camino a la nueva recepción de lo fantástico en la literatura $^{2}$.

Palabras claves: Fantasmagénesis en el siglo XX, Literatura fantástica en Europa (Alemania), Lachmann, Wünsch.
\end{abstract}

Ricarda Hirte

Resumo: A tradição de oferecer uma explicação teórica ao fantástico tem na literatura uma longa trajetória desde a Antiguidade, quando Aristóteles e Platão já tinham refletido sobre o tema. Pode-se dizer, no entanto, que com os escritos de Tzvetan Todorov essa tradição chega a seu ponto central, uma vez que as teorias antigas culminam no texto de Todorov e as novas derivam dele. No âmbito das culturas de língua alemã e francesa pode-se observar, dede então, uma discussão viva com o objetivo de oferecer um referencial teórico ao fantástico na literatura para poder então definir a própria literatura fantástica que teve seu auge na Alemanha do final do século XIX. A contribuição de Marianne Wünsch, que se discute e se apresenta em seguida, contribui com elementos essenciais para abrir caminho para uma nova visão do fantástico na literatura.

Palavras-Chave: Fantasmogênese no século XIX, Literatura fantástica na Europa (Alemanha), Lachmann, Wünsch.

1 Departamento de Mecánica, Cátedra de Idioma Moderno Alemán, Facultad de Ciencias Exactas y Tecnología, Universidad Nacional de Tucumán, San Miguel de Tucumán, Provincia de Tucumán, Argentina; Doctora Internacional de la Universidad de Valencia, España; especializada en: oralidad y escritura: Lenguajes especializados en el ámbito anglogermánico; grupo de investigación: "Recreación ficcional de la experiencia en la narrativa literaria y en la autoficción", Universidad Nacional de Tucumán (CIUNT). Correo electrónico: r.hirte@gmail.com.

2 Vease: HIRTE, Ricarda. Eine Lesart der Golem-Figur bei G. Meyrink und P. Wegener. San Miguel de Tucumán: Universidad Nacional de Tucumán, 2013. 
La discusión de lo fantástico o digamos la ansiedad de dar imagen teórico a lo fantástico conocemos desde la edad antigua. Aristóteles intenta definir el concepto y su derivación de lo fantástico en su tratado memoria et reminiscentia y asocia con el concepto phantasma una imagen genuina e ilusoria simultánea. Su idea fue adoptada por Platón pero él lo aplica a la pintura y nombra al concepto phantasia justificándose que para la visualización de la imagen genuina e ilusoria de Aristóteles se necesita la fantasía. Son solamente dos ejemplos brevemente mencionados para darnos una idea que el concepto posee una larga trayectoria. De tal manera la tradición antigua se enlaza con el Concettismo y la estética del siglo XVIII a través de hilos conceptuales, los cuales juegan un papel importante en la generación de la discusión de lo fantástico en el siglo XX.

A continuación la mirada de la discusión de lo fantástico del siglo XX se concentra en Europa y sobre todo en los países de Francia y Alemania que aportaron a la discusión del concepto los impulsos e enfoques innova- dores que oscilan alrededor de la siguiente pregunta principal: ¿Cuáles son los elementos característicos de la literatura fantástica? Teniendo en cuen- ta la pregunta se explica lo expuesto en adelante que no solo quiere dar una respuesta a la misma sino que quiere realizar una breve fantasmagéne- sis del siglo XX.

En el centro de la discusión de lo fantástico del siglo XX se hallan las teorías de Roger Caillois, Louis Vax y Tzvetan Todorov; mientras Todorov necesita de una observación más intensa, porque todos los nuevos emprendimientos de la discusión recurren a él. Pero quiénes prepararon la base de una discusión de lo fantástico en el siglo pasado eran los propios autores de la literatura fantástica y sus críticos. Sobre todo la obra Das öde Haus ${ }^{3}$ de E. T. A. Hoffman influenció mucho la discusión. La novela empieza con la afirmación de Lelios: "Man war darüber einig, dass die wirklichen Erscheinigungen im Leben oft viel wunderbarer sich gestalten, als alles, was die regste Fantasie zu erfinden trachte" (HOFFMAN, p. 139) ${ }^{4}$ para poder

3 HOFFMANN, E. T. A. Das öde Haus. In: Nachtstücke. München: dtv, 1984, p. 139-144. Las citas del texto se hallan en el idioma original. Si hace falta para la comprensión del texto una traducción, la misma fue realizada por la autora del presente texto sin carácter oficial.

4 Traducción: Ellos estaban de acuerdo que las apariencias de la vida se presentan aún más maravillosas que la fantasía más viva pueda inventar.

140 Número temático: Vertentes do insólito nas literaturas das Américas. A Cor das Letras UEFS, n. 15, 2014 
empezar con Franz una conversación sobre un supuesto don profético. Pero el protagonista Theodor de la novela da al lector finalmente una definición de lo extraño y de lo maravilloso, antes que este narra a sus amigos un suceso que le ocurrió hace poco y que afirma la definición de lo fantástico del protagonista y del autor Hoffman:

Aus Eberhards Synonymik mußt du wissen, das wunderlich alle Äußerungen der Erkenntnis und des Begehrens genannt werden, die sich durch keinen vernünftigen Grund rechtfertigen lassen, wunderbar aber dasjenige hei $\beta t$, was man für unmöglich, für unbegreiflich hält, was die bekannten Kräfte der Natur zu übersteigen, oder wie ich hinzufüge, ihrem gewöhnlichen Gange entgegen zu sein scheint. [...]

Aber gewi $\beta$ ist es, da $\beta$ das anscheinend Wunderliche aus dem Wunderbaren sproßt, und das wir nur oft den wunderbaren Stamm nicht sehen, aus dem die wunderlichen Zweige mit Blättern und Blüten hervorsprossen (HOFFMAN, p. 140-141) $)^{5}$.

Hoffman da aquí dos variantes en las cuales puede manifestarse lo fantástico, lo extraño y lo maravilloso. Ambas variantes pertenecen al ámbito de lo inexplicable. El intento de Hoffman de explicar las posibles expresiones de lo fantástico formula la base de la teoría de Roger Caillois. En las escrituras filosóficas analiza Caillois lo no lógico con ayuda de métodos lógicos. Sobre todo en las dos obras Au cœur du fantastique ${ }^{6}$ y Das Bild des Phantastischen. Vom Märchen bis zur Science fiction ${ }^{7}$, Caillois desarrolla su definición para explicar lo fantástico. Generalmente Caillois se basa en que lo fantástico irrumpe el orden en vigor, es decir, que en la legitimidad invariable de la cotidianidad irrumpe lo ilícito: „le fantastique est rupture de l'ordre reconnu, irrumtion de l'inadmissible au sein de l'inaltérable légalité quotidienne, et non substitution totale a l'univers réel d'un univers exclusivement miraculeux" (CAILLOIS, p. 161). Entonces lo fantástico no substituye

5 Traducción: Debes saber de la sinonímica de Eberhard que sean llamadas todas las expresiones del conocimiento y del afán extraño que no se justifican por una razón razona ble pero se llama lo maravilloso que sea imposible, inentendible que supera las fuerzas conocidas de la naturaleza o, como yo adjunto, que sean en contra de la dirección habitual. Pero seguro es que el aparente extraño creció del maravilloso y que solamente muchas veces no vemos el tronco maravilloso del cual crecieron las ramas con las hojas y flores extrañas.

6 CAILLOIS, Roger. Au cœur du fantastique. Paris: Gallimard, 1965.

7 CAILLOIS, Roger. Das Bild des Phantastischen. Vom Märchen bis zur Science fiction. Rein A. Zondergeld (Hrsg.). Phaicon I. Frankfurt am Main: Suhrkamp, 1974, p. 44-83. 
el universo real sino irrumpe la realidad del mundo cotidiano sin modificarle. Surge que:

zum Wesen der Phantastik die Erscheinung: was nicht eintreten kann und trotzdem eintritt, zu einer ganz bestimmten Zeit, an einem ganz bestimmten Ort, im Herzen einer bis ins kleinste Detail festgelegten Welt aus der man das Geheimnisvolle für immer verbannt geglaubt hatte (CAILLOIS, p. 50).

La teoría de Caillois insiste frecuentemente en la violación de las leyes que se refleja sobre todo en las palabras "I'inadmissible", para lo inadmisible y "I'indicible" para lo indecible. Su teoría se basa en la realidad de lo cotidiano y ambos conceptos recién mencionados están en oposición. En el trabajo Das Bild des Phantastischen. Vom Märchen bis zur Science fiction Caillois hace referencia al concepto de lo maravilloso que ya fue utilizado por Hoffman. Pero Caillois ve en lo maravilloso no la explicación de Hoff- man que contiene lo imposible e inconcebible sino, lo maravilloso represen- ta para Caillois solamente un mundo de cuento. El cuento, a su vez, es una añadidura al mundo cotidiano, sin tocar o destruir su contexto. Por consiguiente Caillois excluye lo maravilloso de su teoría de lo fantástico y le da una asignatura propia, dónde se revela en el cuento.

Los conceptos de Caillois de lo inadmisible e indecible están compensados en la teoría de Louis Vax ${ }^{8}$ por el concepto del "I'inexplicable". Lo inexplicable se refiere a lo inexplicable racional y se discute en el conflicto entre real e irreal. Para Vax "le fantastique et le feerique (son) deux espèces du genre merveilleux" e especifica lo fantástico como "fille de l'incroyance" ${ }^{\prime \prime}$. La teoría de Vax no está tan elaborada como la de Caillois a pesar que no diferencia entre lo maravilloso y lo fantástico. En la argumen- tación de Vax existe una coexistencia de los tres conceptos "fantastique", "féerique" y "merveilleux" sin definir sus límites. Esto último lo intenta Pier- re Georges Castex:

Le fantastique est essentiellement intérieur et psychologique, il ne se confond pas avec l'affabulation des récits mythologiques ou des féeries qui

8 Véase VAX, Louis. La séduction de l'étrange. Etude sur la littérature fantastique. Paris, 1965.

9 VAX, Louis. L'art et la littérature fantastique. Que sais-je?, n. 907, Paris, P.U.F., 1974, p. 72.

142 Número temático: Vertentes do insólito nas literaturas das Américas. A Cor das Letras UEFS, n. 15, 2014 
impliquent un dépaysement de l'esprit [...] il se caractérise au contraire par une intrusion brutale du mystère dans le cadre de la vie réelle ${ }^{10}$.

Si uno intenta a esta altura de lo dicho, encontrar similitudes entre las teorías, surge que lo fantástico siempre está como si fuera un elemento que irrumpe el mundo cotidiano y que destruye el orden de este mundo.

Con Tzevetan Todorov la discusión alrededor de lo fantástico recibe nuevos aspectos, y será indicativo para el último tercio del siglo XX. La explosión inicial de la Introduction à la littérature fantastique era según Lachmann ${ }^{11}$ Vladimir Solov'ev quien desarrollo en 1895 una formula general. Solov'ev se basa en que el mundo está dividido en dos: esta vida (aquende) y el más allá (allende). La mística forma parte del allende y penetra imperceptible el aquende. Precondición es que la realidad de los mundos vividos opera como base para reconocer las apariciones del otro. El otro importuna al mundo cotidiano en su conjunto e implica otra causalidad para explicarse.

Andere 'Kausalität' heißt, daß die Regeln der Logik suspendiert sind und Tiefenbedeutung die Oberflächenbedeutung der lebensweltlichen Ereignisse überdeckt. Im Aufdecken dieser Tiefenbedeutung erklärt das Phantastische seine eigentliche Funktion (LACHMANN, p. 89).

Un carácter transcendental no se puede negar en la teoría de So- lov'ev porque lo fantástico ocupa una posición umbral entre la vida y el más allá; posición umbral en el sentido que lo fantástico se refiere al más allá, la mística, y que se manifiesta en el momento cuando importuna al mundo cotidiano.

Todorov mismo hace referencia al concepto de lo fantástico de Solov'ev en la Einführung in die fantastische Literatur $^{12}$ :

Es gibt eine unheimliche Erscheinung, die man auf zweierlei Weise erklären kann, nämlich entweder aus natürlichen Ursachen oder aber aus übernatürlichen. Die Möglichkeit der Unschlüssigkeit angesichts dieser Alternative schafft die Wirkung des Fantastischen (TODOROV, p. 26).

En la tensión entre "natural" y "sobrenatural" aparece lo fantástico en la indecisión, en el momento cuando una persona se ve enfrentado a un

\footnotetext{
10 CASTEX, Pierre George. Le conte fantastique en France de Nodier à Maupassant. Paris, 1951, p. 10/11.

11 LACHMANN, Renate. Erzählte Phantastik. Frankfurt am Main: Suhrkamp, 2002, p. 89.

12 TODOROV, Tzvetan. Einführung in die fantastische Literatur. Walter Höllerer. (Hrsg.). München: Hanser, 1972.
} 
suceso, el cual debe clasificar en "verdadero" o "falso" o respectivamente en "natural" y "sobrenatural". En este punto se debe anotar que Todorov implica en su teoría a un lector que será confrontado con un texto narrativo y al cual debe valorar. Este concepto describe primeramente la definición rudimentaria de lo fantástico de Todorov: „Das Fantastische ist die Unschlüssigkeit, die ein Mensch empfindet, der nur die natürlichen Gesetze kennt und sich einem Ereignis gegenübersieht, das den Anschein des Übernatürlichen hat" (TODOROV, p. 26). De este enfoque desarrolla Todorov su esquema de cinco clases que separa lo fantástico de los conceptos emparentados con lo extraño (étrange en la versión francesa) y de lo maravilloso (merveilleux en la versión francesa).

Lo extraño puro y lo fantástico-extraño conducen al lector implícito, en cuanto a la aclaración del suceso inexplicable del texto, hacia lo extraño, mientras lo fantástico-maravilloso y maravilloso-puro dirigen hacia lo maravilloso. Lo extraño se caracteriza porque es explicable, es decir aunque se trata de un producto de la imaginación o de una alucinación que parece ser fuera de la ratio, el suceso es explicable con la ayuda de las leyes del mundo real en el cual se mueve el lector implícito. Lo maravilloso al contrario no busca una aclaración porque se halla fuera de la ratio y se supone a un lector que acepta la legitimidad sobrenatural. Lo fantástico (fantástico-puro) se encuentra cuando el lector implícito no puede resolver el suceso con normas que dirigen hacia lo extraño ni hacia lo maravilloso. La vacilación del lector persiste sin ser resuelta.

Lo fantástico de Todorov está sujeto por características estructurales de donde se derivan las funcionales. De tal manera, lo fantástico como género está atado a tres condiciones: la primera es la vacilación del lector, es decir, que, como precondición general se supone una bidimensionalidad del mundo, cuyos polos se hallan en "lo natural" y en "lo sobrenatural". Además, el lector debe contraer un pacto ficticio dónde el mundo literario es igual al mundo del lector; la segunda condición es que la vacilación debe ser notada también de la persona actuando, el lector se somete a una regla de identificación, y la tercera el lector debe "leer", es decir que éste no debe aplicar al texto una interpretación alegórica o poética. Estas tres condiciones estructurales precisan y completan la definición rudimentaria de lo fantástico que Todorov explicita de tal manera:

Zuerst muß der Text den Leser zwingen, die Welt der handelnden Personen wie eine Welt lebender Personen zu betrachten, und inn unschlüssig werden lassen angesichts der Frage, ob die evozierten Ereignisse einer natürlichen 
oder einer übernatürlichen Erklärung bedürfen. Des weiteren kann diese Unschlüssigkeit dann gleichfalls von einer handelnden Person empfunden werden; so wird die Rolle des Lesers sozusagen einer handelnden Person anvertraut und zur gleichen Zeit findet die Unschlüssigkeit ihre Darstellung, sie wird zu einem Thema des Werkes; [...] Dann ist noch wichtig, daß der Leser in bezug auf den Text eine bestimmte Haltung einnimmt: er wird die allegorische Interpretation ebenso zurückweisen wie die "poetische« Interpretation. Diese drei Forderungen sind nicht gleichwertig. Die erste und die dritte konstituieren tatsächlich die Gattung; die zweite kann auch unerfüllt bleiben. Dennoch erfüllen die meisten Beispiele alle drei Bedingungen (TODOROV, p. 33).

De las características estructurales se derivan las funcionales que Todorov clasifica en tres clases: los temas del yo y los temas del tú. Ambas clases se pueden resumir como funciones sociales y se refieren a temas que son por una parte relacionados con el sujeto y por otra parte objetivan los conflictos del sujeto. La tercera clase se refiere a las funciones literaria, pragmática, semántica y sintáctica de un texto, mientras la cuarta clase analiza la función del texto bajo la perspectiva humanística y científico- literaria.

Con la definición de lo fantástico de Todorov hemos llegado en cuanto a la fantasmagénesis a un punto a dónde acuden las definiciones anteriores y de dónde emprenden las nuevas. Con respeto a lo fantástico se puede decir hasta ahora que las categorías de tiempo, espacio y causalidad están desfiguradas; pues no son explicables con las normas de la convención mimética ni respetan los principios estéticos de la convención y de la semejanza. "Gemä $\beta$ den Modi ihrer Auseinandersetzung mit der Fiktion präsentiert sich Phantastik in drei Formen: als Usurpation der Fiktion, als deren Überschreitung und als Gegenfiktion" (LACHMANN, p. 98) explica Lachmann la presentación de lo fantástico, mientras

die Usurpation der Fiktionsmorphologie und -topik erlaubt, das Phantasma mit Hilfe fiktionaler Parameter zu motivieren. In der Überschreitung wird die Geltung dieser Parameter zwar noch aufrechterhalten, aber überdehnt. Die Phantastik als Gegenfiktion streicht die fiktionalen Parameter (LACHMANN, 98).

Como ya fue contestado hemos llegado a un punto en la fantasmagénisis que explica el concepto de lo fantástico pero se deberían adjuntar otros aspectos que definen más el carácter de lo fantástico. El fantasma, y generalizando lo fantástico, recurre siempre a un contexto cultural. Si se presta a este atributo del fantasma la atención, lo fantástico formula afir- 
maciones antropológicas, es decir, mientras un individuo se apodera de rasgos fantásticos, el agranda hasta data el conocimiento antropológico. Esta extensión se manifiesta en el texto fantástico en forma de sueños, alucinaciones y demencia que experimenta el individuo fantástico. De tal manera entran en el ámbito de la posibilidad transformaciones en entes no reales. Para el protagonista de lo fantástico significa que se halla permanentemente en estados emocionales excéntricos, les debe soportar y/o superar. Pero el conocimiento antropológico siempre está atado al conocimiento cultural. La componente culturológica alberga dos aspectos: cada cultura posee un ámbito de lo reprimido, lo olvidado, el tabú, pues de lo extraño que debe ser excluido según las normas vigentes de la cultura. Una cultura vive de la oposición extraño-propia para establecer un orden cultural. Mi- entras el texto fantástico tematiza lo extraño o no propio de una cultura él recupera bienes culturologicos que han sido víctimas de las exclusiones y provocan igualmente desorden; desorden en el sentido que lo real y con esta palabra se refiere a la presencia de una cultura en función, se cuestio- na. Lo irreal que posee el texto fantástico se roza con las categorías de lo real. Se asocia a este primer aspecto el segundo lo cual caracteriza la base de un texto fantástico y que se define en su momento de creación de la cultura reinante. En otras palabras, un texto de la época de la inquisición que se basa en la existencia de brujas puede ser leído en el siglo XVIII como un texto fantástico aunque reclama en su contexto histórico ser realístico. Debido a que el protagonista de un texto fantástico se pone en contacto con lo irreal o con los temas de una cultura marginarías, lo real como cate- goría está puesto a prueba. Muchas veces el protagonista de estos textos se ve enfrentado con ciencias ocultas y esotéricas en las cuales entran elemen- tos cabalísticos, gnósticos y herméticos. Pues se encuentra con rituales no cristianos o él mismo sea instruido en lo extraño. Pero lo fantástico sola- mente se revela en cuanto a lo real porque lo imposible, lo irreal y lo con- traracional es contrapuntístico a lo posible, lo real y lo racional. Lo fantásti- co narra el encuentro de la cultura con su olvido.

De lo ante dicho se deduce que lo fantástico está atado a una componente cultural la cual representa el sistema actual pensante y que se manifiesta, en cuanto a la literatura, a estructuras textuales. Se debe repetir aquí que la literatura en la cual aparecen demonios, semidioses, brujas, fantasmas etc. tienen una larga historia culturológica y literaria pero no pertenecen a la literatura fantástica en sí, que se localiza a partir del fin del siglo XVIII en el ámbito de habla alemán. Además se puede contestar que 
coincide un florecimiento de la literatura fantástica con una época en la cual las ciencias ocultas tienen relevancia cultural. De la misma manera se trata de los conceptos de lo maravilloso o lo sobrenatural, para referirse a términos de Todorov y Caillois, que pueden aparecer como elementos en un texto fantástico pero que no son típicos para este género de literatura porque están aplicables a otras estructuras textuales, como por ejemplo a cuentos, fabulas etc.

Para destacar el aspecto del componente culturológico se debe hacer referencia a Marianne Wünsch ${ }^{13}$ que integra en su análisis en cuanto al concepto de lo fantástico, aparte de la componente culturológica también la antropológica. Ella no se basa en la historia receptiva del concepto, sino explica y define la literatura fantástica a un nivel textual inmanente. Así se deducen dos maneras para que se represente lo fantástico e incluso lo irreal en un texto fantástico: los fantasmas subjetivos y los objetivos. Se entiende como fantasmas subjetivos todos aquellos que se derivan de sueños, alucinaciones o sucesos parecidos, que produce directo o indirecto el protagonista de lo fantástico. Los fantasmas objetivos a su vez son independientes del protagonista de lo fantástico y son experimentados por otras personas. Estos fantasmas se asocian con sucesos maravillosos o sobrenaturales, que no son provocados por el hombre y que no son expli- cables racionalmente. Ambos tipos de fantasmas unen una característica que se encuentra también en todos los textos teóricos que se ocupen de lo fantástico: lo fantástico se manifiesta „durch das Auftreten von etwas in der dargestellten Welt zustande, für das es in der geglaubten Weltordnung keinen theoretischen Platz gibt, das heißt durch das Auftreten eines „unerklärlichen Außer- oder Übernatürlichen”, das die geglaubte Weltordnung fundamental in Frage stellt" (WÜNSCH, p. 15) ${ }^{14}$. Se trata pues de un suceso que irrumpe en el orden mundial existente o en la presente realidad. El suceso es, como lo expresa Wünsch, atado a un Realitätsbegriff (una traducción sería concepto de la realidad pero es preferible mantener la palabra en su original).

13 WÜNSCH, Marianne. Die fantastische Literatur der frühen Moderne: (1890-1930);

Definition; Denkgeschichtlicher Kontext; Strukturen. München: Fink, 1991.

14 Traducción de la cita: Lo fantástico se manifiesta a través de la presencia de un algo en el mundo presentado para lo cual no haya sitio teórico en el orden mundial actual, es decir a traves de la presencia de "un no explicable extra o sobrenatural", que interroga fundamentalmente el creyente orden mundial. 
El enfoque de Wünsch se basa en el hecho que un texto fantástico no se debe hacer dependiente de la intención de su autor ni de la recepción de un lector, además que no opera a través de un catálogo de motivos o temas que puedan aparecer en la literatura fantástica. Se busca la repuesta en un nivel estructural:

das „Fantastische” kann nicht sinnvoll auf der texttypologischen Ebene, sondern muss auf der Ebene elementarer Strukturen definiert werden: das „Fantastische” ist nicht als Texttyp, sondern es ist als eine vom Texttyp unabhängige Struktur, die als Element in verschiedenen Texttypen und Medien integriert werden kann, einzuführen. Die Klassenbildung „fantastische Literatur" ist dann keine elementare, sondern eine abgeleitete Größe: sie bezeichnet die Texte, in denen das Fantastische dominant ist (WÜNSCH, p. 13).

Este enfoque implica una mirada hacia la definición de estructuras narrativas, porque lo fantástico, como ya dicho, puede aparecer en diversas tipos de textos y está integrado de tal manera en una "historia". Aquí se debe mencionar la definición de Jurij M. Lotman lo que es una estructura narrativa porque cuenta en un contexto textual como cultural.

Según Lotman hay una estructura narrativa cuando hay un suceso. El suceso a su vez está caracterizado por la Grenzüberschreitung (extralimitación), lo que significa que un apoderado, o sea lo que en este contexto es un portador de la acción, traspasa las limitaciones de dos espacios semánti- cos. El espacio semántico está definido por un orden que está reconocido por el apoderado como normal en cada contexto textual específico. El or- den consiste de rasgos semánticos como regularidades, normas, localida- des. Deja un apoderado este orden y entra en otro espacio semántico que está descripto en el texto, el cual traspasa una limitación. El nuevo espacio semántico posee un sistema de órdenes propios, puede, pero no tiene que poseer rasgos del primer espacio, lo determinante es que el nuevo espacio posee un orden que no es reconocido por el apoderado como normal. Sin embargo es irrelevante si el orden "normal" puede existir fuera del texto, pues solamente se lo puede verificar con el texto. Se deduce que ambos espacios semánticos están en oposición. Para el apoderado significa que se mueve entre dos sistemas de órdenes y en cuanto a lo fantástico que se 
mueve en un mundo real e irreal. El suceso es indiferente al género y puede aparecer en distintas estructuras narrativas ${ }^{15}$.

En cuanto a lo fantástico la definición de Lotman aporta mucho porque se integró una variable que permite estudiar el suceso en distintos contextos. „Das heißt ein - und derselbe Sachverhalt kann je nach Text bzw. je nach kulturellem System der Epoche in einem Falle ein Ereignis, im anderen ein Nicht-Ereignis sein" (WÜNSCH, p. 15). En el caso de lo fantástico significa que se trata de un fenómeno a nivel de la histoire y que es independiente del discurso de las figuras en el texto. Implica además que solamente un suceso en el texto puede convertirle en un fantástico y para que sea evaluado el suceso como un fantástico se necesita según Wünsch una Historizitätsvariable (una variable de la historia o de una posible conexión con la historia para reflejar más o menos el significado del término utilizado en el original) que sea idéntico con el Realitätsbegriff. En él está connotado el saber cultural de una época. En el saber cultural se hallan afirmaciones que van desde el llamado conocimiento cotidiano hasta el conocimiento científico y no tiene importancia si estas afirmaciones forman parte de la creencia o del conocimiento y si estas afirmaciones son verdaderas o falsas; lo que importa en este contexto es que la época está convencida de la veracidad de estas afirmaciones.

Entonces si un suceso desata lo fantástico e irrumpe en un orden actual, lo que significa más específico que pone en duda el Realitätsbegriff, y que se puede suponer que lo fantástico nunca tendrá según las teorías actuales un sitio teorético en el orden, pues que sea inexplicable, se puede manifestar este suceso como un extra o sobrenatural en forma de hechos o como un ente. Pero cada suceso que se refiere a hechos o a un ente, entonces que será fantástico, necesita una explicación que abarca el mismo texto. Puesto que se supone que haya un clasificador de la Realitätskompatibilität (la compatibilidad de la realidad) en el texto fantástico se deduce que explícito o implícito se determina la Erklärungsbedürftigkeit (la necesidad de una explicación) del elemento fantástico en el texto; es decir con otras palabras que el elemento fantástico aparece en el margen del conocimiento cultural como inexplicable, considerando que generalmente cada cultura acepta a

Como ampliación: se refiere con otras estructuras narrativas a textos narrativos en general pero también al drama como al film. Enfilar distintos sucesos se forma la histoire, la cual se modifica a través del discours. Debido a las características del medio y del género como la interpretación si lo aplicamos al teatro o al film, se evoca un significado diferente de la histoire. 
fenómenos que son inexplicables pero que sean tomado por la cultura como hechos reales. Estos fenómenos no explicables no son fantásticos. El suceso fantástico entonces es inexplicable e incapaz de explicarse, lo que contiene que no sea explicable en un momento más adelante. Debido a que este suceso aparece dentro de un texto que por sí recurre a un Realitätsbegriff, debe haber dentro del texto la posibilidad del cuál reconoce al suceso fantástico como real. Esta posibilidad aporta en la mayoría de estos textos el protagonista que no solamente percibe al fenómeno sino lo califica como real o no real. Wünsch lo expresa de la siguiente manera:

[...], dass irgendeine Figur der dargestellten Welt zu irgendeinem Zeitpunkt in der erzählten Geschichte das Phänomen nicht als nicht-real setzt, d.h. zumindest seine Möglichkeit ernstlich erwägt, wobei diese Figur offenkundig die Bedingung erfüllen muss, vom Text als zurechnungsfähig klassifiziert zu werden (WÜNSCH, p. 44).

El problema de la explicación se deriva del hecho que el fenómeno no está percibido en la ficción poética como un no real. Jaques Finné ${ }^{16}$ integró en su trabajo esta necesidad de la explicación que Todorov solamente roza. Finné consta que „tout recit fantastique est subordonne à une explication” (Finné, 36). Finné además menciona todos aquellos casos de la literatura fantástica que el fenómeno no se explica o mejor dicho que el suceso no recibe una explicación. Lo fantástico entonces no implica una explicación pero recurre por supuesto a una estructura explicativa dentro de la ficción poética. Esta estructura explicativa no se entiende como una científica teórica sino expresa que hechos inexplicables reducen a otros hechos (los cuales puedan ser en un extremo también inexplicables). Estos hechos están llevados en texto por el discurso de una figura o figuras o de un ente narrativo que están a su vez atados a su conocimiento cultural y a su Realitätsbegriff. Aunque se supone que casi todos los textos dan indicios hacia una explicación hay casos en los cuales los fenómenos quedan inexplicables. Esta pura inexplicación remite al ámbito de explicaciones ocultas: „denn diese sind die einzige Erklärungsklasse, die fantastische Ereignisse kennt und die zugleich dem kulturellen Wissen, zumindest als Wissen über die Existenz solchen angeblichen Wissens, bekannt ist" (WÜNSCH, p. 47).

Siguiendo el hilo argumentativo de Wünsch, la explicación del fenómeno se reparte en dos clases: las explicaciones conformes con el conoci-

16 FINNE, Jacques. La littérature fantastique. Essai sur l'organisation surnaturelle. Bruxelles, 1980.

150 Número temático: Vertentes do insólito nas literaturas das Américas. A Cor das Letras UEFS, n. 15, 2014 
miento y las explicaciones no conformes con el conocimiento. Las explicaciones conformes con el conocimiento contienen todos aquellos enfoques explicativos que estén conformes con el conocimiento cultural; lo que significa que el fenómeno pone en duda el Realitätsbegriff por un tiempo porque fue malentendido o mal interpretado por la figura percibida. Se trata por un lado de la negación de datos aportados por los sentidos y por otro lado de elementos explicativos a los cuales recurre el fenómeno. Este caso se manifiesta si un fenómeno es explicado a través de alucinaciones o de sueños y clasificado como no real o si datos aportados por los sentidos sean malinterpretados. Este último caso por ejemplo se manifiesta si la figura percibida insinúa a sí mismo o fue insinuado el fenómeno, pues si se trata de un engaño o autoengaño. Las explicaciones conformes con el conocimi- ento psicológizan o respectivamente patologizan o criminalizan al fenóme- no. Aquí son mencionados ejemplos como la Gothic Novel como algunas novelas criminalísticas del siglo XX.

Las explicaciones no conformes con el conocimiento se resumen en el ocultismo, lo que significa que el fenómeno es un suceso potencialmente oculto que recibe su existencia a través del texto partiendo de su real existencia. Wünsch se basa en el hecho que un suceso oculto es un fenómeno que será interpretado, en cuanto al Realitätsbegriff cultural, como un suceso imposible e inexplicable pero que manifiesta su realidad dentro de la ficción poética. Según lo dicho es una explicación ocultista

die nicht nur die Existenz okkulter Phänomene akzeptiert, sondern zudem diese unter Rekurs auf Annahmen „erklärt”, die ihrerseits selbst fundamentale Basispostulate des Realitätsbegriffs, vermutlich insbesondere theologische Basispostulate, verletzen, indem sie die Existenz von Kräften und Wesenheiten ansetzt, die das kulturelle Wissen ausschließt (WÜNSCH, p. 49).

Con otras palabras significa la cita que si un texto se decidió por una explicación no conforme con el conocimiento, el texto no tiene que ofrecer una alternativa que implica una explicación. A la oposición están todos aquellos textos que se basan en explicaciones conformes con el conocimiento. Pero como la aparición del fenómeno no es descifrado en un primer momento con las herramientas de la explicación conforme con el conocimiento se debe tener en cuenta la posibilidad de una explicación no conforme con el conocimiento. El fenómeno incompatible con la realidad irrumpió al conocimiento cultural y busca una explicación. Una posible explicación ofrece el ámbito del no conforme con el conocimiento que implica en espe- 
cial explicaciones que se hallan en el ámbito ocultico. De tal manera entra al conocimiento cultural un conocimiento reprimido y desconocido que al principio del texto será no conforme con el conocimiento y recibe su explicación a lo largo del texto.

En la literatura fantástica, entre 1890 y 1930, tenemos sobre todo el caso recién descripto que los fenómenos serán explicables con explicaciones no conformes con el conocimiento. Generalmente no exige esta clase de explicaciones que ofrece una alternativa conforme con el conocimiento porque se hallan fuera del conocimiento cultural y no ponen en duda el Realitätsbegriff de cada cultura. Mientras su explicación indica al desconocimiento o la existencia de "mundos superiores", el suceso fantástico puede quedarse en su totalidad inexplicable si aspectos parciales del fenómeno reciben una aparente explicación dentro de la ficción poética.

La existencia de fenómenos no compatibles con la realidad implica que deben existir entidades en el conocimiento cultural (entes o fuerzas) no previstos y que se supone un "mundo superior" ontológicamente distinto al conocido, superando la realidad empírica. En este punto, Wünsch introduce en su argumentación el concepto de mitología que es un sinónimo para la presentación de un "mundo superior", el cual está excluido del conocimiento cultural pero aceptado por la teoría ocultista. Si un texto da una explicación ocultista de uno o varios fenómenos, el texto se remite al texto a una mitología sistemática que no debe ser mencionado en el texto. Resulta que puede existir una discrepancia entre una mitología exigida por el texto y presentada o dada. Con otras palabras, si el texto da el esquema de la mitología utilizada y explica el fenómeno, el mismo sería integrado en el conocimiento cultural y pasa de ser un fenómeno a un suceso real. Interesante es que el apogeo de la literatura fantástica calle en épocas en las cuales el conocimiento oculto es relevante. Se puede derivar que una condición de lo fantástico se da cuando en la cultura actual importa el conocimiento oculto. Literatura fantástica de otras épocas juegan un papel inferior porque están atados al sistema cultural de su época de creación. El conocimiento oculto se comporta distinto porque viene de épocas y sistemas culturales anterio- res y es transpuesto en el espacio temporal relevante dónde recobra de nuevo vida. Este conocimiento oculto se modifica según la cultura en la cual aparece nuevamente en un sentido que sea aceptado por el Realitätsbegriff del ocultismo actual. De tal manera, fenómenos se pueden explicar con la ayuda del conocimiento oculto que está conforme con la propia época. Wünsch menciona aquí que las entidades ocultas que no son aceptables 
culturalmente funcionan como signos de entidades ocultas aceptables. Se deriva una segunda condición por la aceptación de fenómenos fantásticos: si aparecen fenómenos fantásticos en un texto que no se basan en el conocimiento oculto, puedan ser aceptados por la cultura actual y su Realitätsbegriff cuando „menschliche Wahrnehmungsmöglichkeit bedingte uneigentliche Zeichen einer okkulten und akzeptablen Wirklichkeit gelten" (WÜNSCH, p. 59) (cuando ellos valen como señales de una realidad oculta y aceptables dentro de las posibilidades de la percepción humana). Wünsch menciona en este contexto una tercera condición que legitima lo fantástico. Debido a que existen textos que poseen una estructura fantástica pero carecen de una mitología explicativa y recurren a un sistema oculto, pero que están aceptados por la cultura, debe haber un aspecto adicional: este se halla en la naturaleza de la literatura. Literatura fantástica es sobre todo en un primer momento literatura y significa que se puede interpretar y lleva significados. Para los sucesos fantásticos significa que serán aceptados por la cultura si consciente- o inconscientemente simbolizan, en cuanto a su posible interpretación, ideologismos de la época. Estos sucesos están vinculados con significados interpretativos y recurren a un contexto metafórico de la época implícita - o intuitivamente conocido para articular miedos y deseos.

Formulando la contestación a la pregunta inicial ¿qué son los elementos característicos de la literatura fantástica? surge como resultado, que lo fantástico aparece en una estructura narrativa en distintos géneros literarios y medios. Dentro de la misma hay una figura que percibe un fenómeno y una instancia del texto que intenta dar explicación al mismo; mientras el fenómeno por sí mismo puede llevar elementos de la explicación. El fenómeno, pues, no es compatible con la realidad, es decir que la cultura actual no lo percibe como un algo realmente existente; se debe añadir que la aparición del fenómeno le acerca a una posible realidad y su explicación será en el ámbito oculto. El texto debe aportar a una instancia que opera como un clasificador de la incompatibilidad de la realidad y lo manifiesta implícito o explícito. Además, el texto no debe contener indicadores que pueden traducir el fenómeno alegóricamente, parabólicamente o simbólicamente. Por consiguiente se presenta un texto fantástico si aparte de la estructura fantástica se verifica lo fantástico en la explicación del fenómeno. 


\section{REFERÊNCIAS}

CAILLOIS, Roger. Au cœur du fantastique. Paris: Gallimard, 1965.

CAILLOIS, Roger. Das Bild des Phantastischen. Vom Märchen bis zur Science fiction. Rein A. Zondergeld (Hrsg.). Phaicon I. Frankfurt am Main: Suhrkamp, 1974, p. 44-83.

CASTEX, Pierre George. Le conte fantastique en France de Nodier à Maupassant. Paris, 1951.

FINNE, Jacques. La littérature fantastique. Essai sur l'organisation surnaturelle. Bruxelles, 1980.

HIRTE, Ricarda. Eine Lesart der Golem-Figur bei G. Meyrink und P. Wegener. San Miguel de Tucumán: Universidad Nacional de Tucumán, 2013.

HOFFMANN, E. T. A. Das öde Haus. In: Nachtstücke. München: dtv, 1984, p. 139-144.

LACHMANN, Renate. Erzählte Phantastik. Frankfurt am Main: Suhrkamp, 2002.

TODOROV, Tzvetan. Einführung in die fantastische Literatur. Walter Höllerer. (Hrsg.). München: Hanser, 1972.

VAX, Louis. La séduction de l'étrange. Etude sur la littérature fantastique. Paris, 1965.

VAX, Louis. L'art et la littérature fantastique. Que sais-je?, n. 907, Paris, P.U.F., 1974.

WÜNSCH, Marianne. Die fantastische Literatur der frühen Moderne: (18901930); Definition; Denkgeschichtlicher Kontext; Strukturen. München: Fink, 1991. 